\title{
Fabrication and Characterization of a Modified Conjugated Molecule-Based Moderate-Temperature Curing Epoxy Resin System
}

\author{
Liu Ding ${ }^{1 *}$, Ji Ping Yang ${ }^{2}$, Xiao Li Hao ${ }^{1}$ and Ting Tong ${ }^{1}$ \\ ${ }^{1}$ National and Local Joint Engineering Laboratory for Slag Comprehensive Utilization and Environmental Technology, School of \\ Materials Science and Engineering, Shaanxi University of Technology (SNUT), Hanzhong, China, ${ }^{2}$ Key Laboratory of Aerospace \\ Advanced Materials and Performance, Ministry of Education, School of Materials Science and Engineering, Beihang University, \\ Beijing, China
}

OPEN ACCESS

Edited by:

Guilherme Mariz de Oliveira Barra Federal University of Santa Catarina,

Brazil

Reviewed by: Philippe Boisse, Institut National des Sciences Appliquées de Lyon, France Mehdi Derradji,

Polytechnic School of Algiers,

Algeria

Bluma Guenther Soares, Federal University of Rio de Janeiro,

Brazil

${ }^{*}$ Correspondence: Liu Ding

dingliu@snut.edu.cn

Specialty section:

This article was submitted to Polymeric and Composite Materials,

a section of the journal

Frontiers in Materials.

Received: 02 July 2020

Accepted: 24 August 2020

Published: 14 September 2020

Citation:

Ding L, Yang JP, Hao XL and Tong $T$

(2020) Fabrication and

Characterization of a Modified

Conjugated Molecule-Based

Moderate-Temperature Curing Epoxy

Resin System.

Front. Mater. 7:577962.

doi: 10.3389/fmats.2020.577962
A 2-Methylimidazole derivative [ethyl glycidyl ether (EGE)-2-MI] was synthesized through the reaction of EGE with the conjugated molecule 2-MI. The structure was confirmed by Fourier transform infrared spectroscopy, ${ }^{1} \mathrm{H}$-nuclear magnetic resonance spectroscopy, and differential scanning calorimeter. The synthesized EGE-2-MI was applied in epoxy resin E-51 as a latent curing agent. Kinetics of the curing reaction were studied by a differential scanning calorimeter method, optimum curing process parameters were determined, storage life of the resin system at room temperature was evaluated, and mechanical properties of the moderate-temperature-cured products were also tested and analyzed. The results revealed that the epoxy resin system using EGE-2-MI as the curing agent can be efficiently cured at $110-120^{\circ} \mathrm{C}$, with an activation energy of $86.34 \mathrm{~kJ} / \mathrm{mol}$. The storage period of the EGE-2-MI/cyanate resin system can reach 14 days under ambient temperature. In addition, the cured mixture with EGE-2-MI/cyanate resin has a tensile strength of $40 \mathrm{MPa}$ and an $\mathrm{Al}-\mathrm{Al}$ adhesion shear strength of $20.11 \mathrm{MPa}$.

Keywords: conjugated molecule, epoxy resin, curing agent, moderate-temperature curing, methylimidazole

\section{INTRODUCTION}

Moderate-temperature-cured epoxy resin systems can be cross-linked at the temperature range of $100-120^{\circ} \mathrm{C}$. Due to the moderate curing temperature and relatively high adhesive strength, this type of adhesive system has extensive usage in many fields, such as aerospace, automobile, medical devices, and mechanical devices (Baroncini et al., 2016; Mozaffari et al., 2016).

Among all kinds of curing agents that can be used in epoxy resin systems, a conjugated molecule, imidazole and its derivatives have some distinguishing properties (Ham et al., 2010; Leena et al., 2017). They have extremely high reactivity in chain polymerization with epoxy resin, superior heat resistance, low tensile elongation, and outstanding adaptability for molecular design, which opens up the possibility of altering the nucleophilic and base-catalyzed processes during polymerization (Unruh et al., 2011; Arimitsu et al., 2015; Zhao et al., 2019). The only but significant drawback of imidazole that may limit its application is the difficulty to control the high reactivity in the chain polymerization. A liquid mixture of imidazole and epoxy resin would convert to insoluble crosslinked solid rapidly even at storage under ambient condition (Maka et al., 2012). Recently, Zhang's group introduced some conjugated materials into epoxy coating, such as diketopyrrolopyrrole, benzodifuronone, and benzodipyrrolidone, which 
resulted in a functional coating system, for instance, the anti-UV or color-changing function (Zeng et al., 2018; Zeng et al., 2019; Zhang et al., 2020).

The conjugated molecule 2-methylimidazole (2-MI) is one of the most common imidazole-based curing agents. It has a curing temperature $\left(80-120^{\circ} \mathrm{C}\right)$ that is suitable for most manufacturing processes. However, due to the excessively high reactivity, the storage life of 2-MI epoxy systems is short, and it only takes 3 days for the mixture to cross-link spontaneously under ambient temperature (Lee et al., 2011). However, in the practical industry such as aerospace and automobile manufacturing, the operation periods are around 10 days, which makes it difficult for 2-MI systems to be utilized. Therefore, suitable modification is crucial for these conjugated molecule curing agents, and considerable research have been conducted on improving the storage stability of conjugated molecule imidazole curing agents (Wong et al., 2007; Fang et al., 2008; Liu and Lu, 2010; Lei et al., 2015; Yang et al., 2018). Arimitsu et al. (2015) synthesized a novel liquid-type thermal latent curing agent using a Michael addition reaction of fumarate with imidazole in $14-53 \%$ yield. The product has a thermally labile bulky long-chain alkyl succinate group at the $\mathrm{N}$ atom on the imidazole ring, which gives the curing agent a decomposition temperature of $280^{\circ} \mathrm{C}$ and a storage life of 10 days. This method could increase the activation energy of the system, and, therefore, prolong the storage time. Despite the decent storage life, however, most of the modified imidazole system is still limited by the high curing temperature.

Herein, a novel glycidyl ether-modified conjugated imidazole derivative ethyl glycidyl ether (EGE)-2-MI was synthesized by decorating the $\mathrm{N}$ atom in imidazole 1-position. The performance in the epoxy curing system was studied and analyzed, aiming to obtain a moderate-temperature latent epoxy curing system with relatively long storage time and large adhesive strength, which can overcome the underlying obstacles facing imidazole-based epoxy systems.

\section{EXPERIMENTAL}

\section{Materials}

2-MI was purchased from Beijing Chemical Co. Ltd., Beijing, China. EGE was purchased from Shenzhen Jiadida New Material Co. Ltd., Guangdong, China. Epoxy resin was mixed with liquid bisphenol-A diglycidyl ether (E-51) purchased from Yueyang Baling Huaxing Petrochemical Co. Ltd., Hunan, China. Cyanate resin (CE) was purchased from Sinopharm Chemical Reagent Co. Ltd., Beijing, China.

EGE (32.8 g, $0.2 \mathrm{~mol})$ and 2-MI (17.24 g, $0.21 \mathrm{~mol})$ were dissolved in ethyl acetate $(150 \mathrm{ml})$, and then the solution was stirred at $85^{\circ} \mathrm{C}$ for $2 \mathrm{~h}$. The reaction mixture was washed with water several times, and the organic solvent was removed by rotary evaporation. Then, $42.03 \mathrm{~g}$ of the solid product was obtained. Based on our previous works (Ding et al., 2017), 2$\mathrm{MI}$ and 2-MI derivative were used as curing agents in epoxy resin systems, and cyanate ester resin (CE) was used as a stabilizer. The curing systems were prepared by E-51 with a certain amount of the curing agent, as shown in Supplementary Table S1.

\section{RESULTS AND DISCUSSION}

\section{Structure of the Ethyl Glycidyl Ether-2-Methylimidazole}

Modification of the conjugated molecule 2-MI by EGE was conducted following a well-established method, as illustrated in Figure 1A. Several molecular characterizations were operated to confirm the modification result of the conjugated molecule. Fourier transform infrared spectroscopy spectra of EGE, 2-MI, and EGE-2-MI are shown in Figure 1B. The absorption peak at $915 \mathrm{~cm}^{-1}$ in the spectrum of EGE can be attributed to the epoxy group. The peaks at 1,068 and $1,539 \mathrm{~cm}^{-1}$ in the spectrum of 2-MI represent the $\mathrm{N}-\mathrm{H}$ bond, which are not found in the spectrum of EGE-2-MI, suggesting $\mathrm{N}-\mathrm{H}$ bonds from 2-MI have reacted with EGE. The peak at $3,117 \mathrm{~cm}^{-1}$ (i.e., vibration of $-\mathrm{OH}$ ) on EGE-2-MI indicates EGE epoxide has a ring opening that reacted with the imidazole group through nucleophilic addition reaction. ${ }^{1} \mathrm{H}$-nuclear magnetic resonance spectra shown in Figure 1C further confirm the structure of EGE-2-MI; after integral calculation analysis of each peak area, the $\mathrm{S}(7.1-7.2): \mathrm{S}(7.01): \mathrm{S}(6.82-6.92): \mathrm{S}(6.71): \mathrm{S}(5.44): \mathrm{S}(3.26-3.38)$ : $\mathrm{S}(2.51)$ ratio is about $2: 2: 2: 1: 1: 1: 2: 2$, matching the theoretical prediction. Furthermore, the narrow endothermic peak along with a single melting point at $187^{\circ} \mathrm{C}$ on the differential scanning calorimeter (DSC) curve (Figure 1D) demonstrating the product is pure. These observations can confirm that EGE-2-MI has been successfully synthesized.

\section{Curing Condition of the Ethyl Glycidyl Ether-2-Methylimidazole Epoxy Resin System}

With the successful modification confirmed, the performance of the novel curing agent needs to be determined. Nonisothermal DSC was conducted to investigate the curing behavior of the EGE-2-MI epoxy resin system (Vyazovkin et al., 2014); the curves for the epoxy resin system examined at different heating rates ranging from 3 to $20^{\circ} \mathrm{C} / \mathrm{min}$ are shown in Figure 2A. With the increase in the heating rate $(\beta)$, the inflection point of the curve moves toward the hightemperature region, and the exothermic peak becomes sharper with increasing area, indicating more heat was released from the curing reaction. From the increasing trend of the initial curing temperature $\left(T_{\mathrm{i}}\right)$, curing peak temperature $\left(T_{\mathrm{p}}\right)$, and final curing temperature $\left(T_{\mathrm{f}}\right)$ with increasing heating rates, it is obvious that in the same curing system, $\beta$ has a significant influence on the characteristic temperatures. To determine the accurate characteristic temperatures of the system under isothermal curing condition, the influence of $\beta$ must be eliminated. In order to achieve that, different characteristic temperatures under different heating rates are recorded and linear-fitted, three near-straight fitting lines are obtained, and the standard characteristic temperatures $\left(T_{\mathrm{i}}, T_{\mathrm{p}}\right.$, and $\left.T_{\mathrm{f}}\right)$ at $\beta=0^{\circ} \mathrm{C} / \mathrm{min}$ are then determined by extrapolation. By plotting $T_{\mathrm{i}}$ against the scanning rate, an equation can be obtained from the fitted line, $T_{\mathrm{i}}=1.114 \beta+98.38$. Treating the fitting equation with the extrapolation method, the value of 

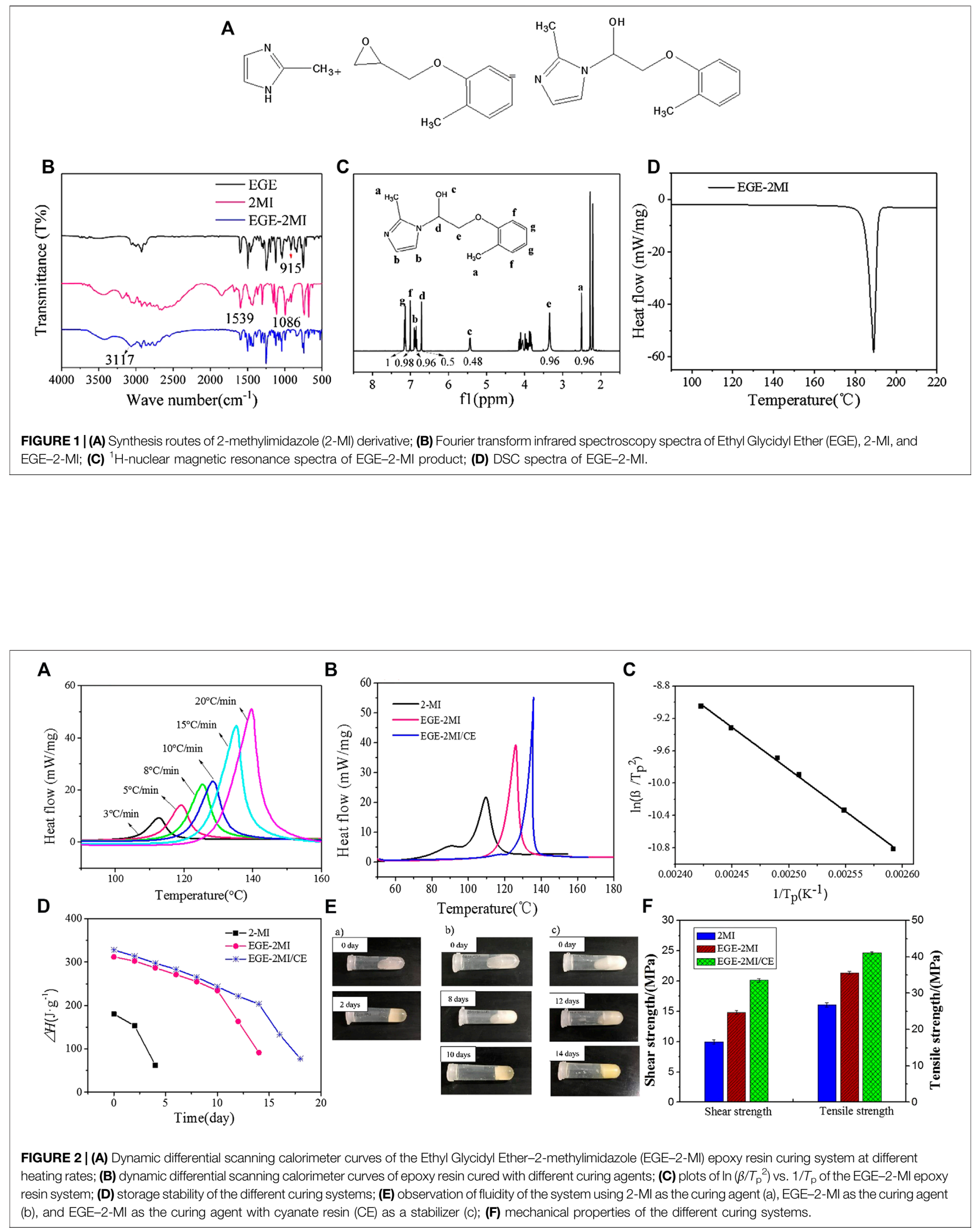
$T_{\mathrm{i}}$ at a scanning rate of $0^{\circ} \mathrm{C} / \mathrm{min}$ can be estimated, which can reflect the curing effect of epoxy resin under isothermal condition. Similarly, the equations of $T_{\mathrm{p}}$ and $T_{\mathrm{f}}$ are $T_{\mathrm{p}}=$ $1.5159 \beta+110.6669$ and $T_{\mathrm{f}}=1.9842 \beta+122.5490$. Therefore, it can be deduced that the optimum curing temperature of the E-51/EGE-2-MI system is between 98 and $110^{\circ} \mathrm{C}$, which can further be cured at $122^{\circ} \mathrm{C}$. Using DSC to analyze the curing behavior of the epoxy resin system under the same heating rate at $10^{\circ} \mathrm{C} / \mathrm{min}$ (Figure 2B), the suitable curing temperature for 2 MI, EGE-2-MI, and EGE-2-MI/CE epoxy systems is 100, 110, and $120^{\circ} \mathrm{C}$, respectively.

\section{Curing Kinetics Analysis}

The study on curing kinetics is helpful to understand the curing process and to optimize curing conditions in order to produce high-quality epoxy systems (Yang et al., 2019). The peak temperature, $T_{\mathrm{p}}$, shifts to a higher temperature region with increasing heating rate, $\beta$. Hence, the Kissinger method can be used to calculate the activation energy and the pre-exponential factor of the curing reaction of epoxy resin examined in this study (Sheng et al., 2008; Dai et al., 2009; Acocella et al., 2016). The equation for the Kissinger method can be expressed as

$$
\begin{gathered}
-\ln \left(\frac{\beta}{T_{p}^{2}}\right)=\frac{E_{a}}{R T_{p}}-\ln \frac{A R}{E_{a}}, \\
\frac{\mathrm{d}\left[\ln \left(\beta / T_{p}^{2}\right)\right]}{\mathrm{d}\left(1 / T_{p}\right)}=-\frac{E_{a}}{R} .
\end{gathered}
$$

Crane:

$$
\frac{\mathrm{d} \ln \beta}{\mathrm{d}\left(1 / T_{p}\right)}=-\frac{E_{a}}{n R},
$$

where $\beta$ is the heating rate $\left({ }^{\circ} \mathrm{C} / \mathrm{min}\right), E_{\mathrm{a}}$ is the activation energy, $T_{\mathrm{p}}$ is the peak temperature, $\mathrm{A}$ is the frequency factor, $\mathrm{R}$ is a gas constant of $8.314 \mathrm{~J} /(\mathrm{mol} \mathrm{K})$, and $n$ is the reaction order. Based on Eqs 1 and 2, through a linear fit between $\ln \beta / T_{p}^{2}$ and $1 / T_{p}$ (Hardis et al., 2013; Ferdosian et al., 2015), the value of activation energy $E_{\mathrm{a}}$ can be obtained from the slope of the fitted line. The $E_{\mathrm{a}}$ of the EGE-2-MI resin can be calculated as $86.34 \mathrm{~kJ} / \mathrm{mol}$, while the apparent activation energy of unmodified 2-MI was $50.34 \mathrm{~kJ} / \mathrm{mol}$ by preliminary experiment (Leena et al., 2017). Activation energy is a measure of the energy required for a curing reaction: the higher the value, the more the energy needed. The increase in activation energy of the modified epoxy resin system reduces the reactivity of epoxy resin in chain polymerization with epoxy resin. The value of pre-exponential factor A can be calculated by reading the value of $\ln \mathrm{A}$ from the $y$-intercept of the fitted line, which is $16.13 \mathrm{~s}^{-1}$ in this case. Based on Eq. 3, the reaction order $n$ can be calculated as 0.927 . The curing kinetic equation is expressed as follows:

$$
\frac{\mathrm{d} \beta}{\mathrm{dt}}=A \exp \left(-\frac{E_{a}}{R T}\right)(1-\beta)^{0.927},
$$

where $n$ is less than 1 , which indicated the curing reaction is then a complex reaction (Xiong et al., 2019).

\section{Storage Stability}

More importantly, the storage life of this modified conjugated molecule curing agent needs to be evaluated to overcome the underlying obstacles of imidazole materials. The storage stabilities of different curing systems were further assessed by measuring the changes in enthalpy values under room temperature. E-51 with 2-MI (system A), EGE-2-MI (system $\mathrm{B}$ ), and EGE-2-MI with $\mathrm{CE}$ (system C) were stored at room temperature $\left(23 \pm 5^{\circ} \mathrm{C}\right)$; enthalpy values of each system were measured and recorded through DSC every 2 days; and the heating rate was $10^{\circ} \mathrm{C} / \mathrm{min}$. Figure $2 \mathrm{C}$ shows the variation of enthalpy value of three systems with increasing storage time. As presented in the plot, the enthalpy value of the 2-MI system drops sharply after storage for 2 days, and the crosslinking degree can be calculated through the variation of the total exothermic heat as $15 \%$; the enthalpy value of the EGE-2-MI system is relatively stable within 10 days and begins to decrease after 12 days, and the crosslinking degree after 10 days is $25 \%$; and the crosslinking degree of the EGE-2-MI/CE system was $25 \%$ after 14 days, and $\mathrm{CE}$ can form a triazine crosslinking network structure at elevated temperature and could decrease the activity of curing agents, which would further extend the storage time.

In addition to the test of enthalpy value of the systems, a more visualized way was used to observe the fluidity of different systems after different storage periods. The same amount of systems A, B, and C was put into three centrifuge tubes, which were placed on the table for a certain storage period, 2, 8 , or 12 days. The tubes were then placed on the table to measure and record the length of the fluid flow. The recording pictures of the three systems are shown in Figure 2E. As demonstrated in the pictures, results of fluidity tests are consistent with those of DSC tests, and fluidity of epoxy resin systems decreases with increasing storage time. The storage period of the 2-MI system is 2 days, which can be apparently improved to 10 days in the EGE-2-MI system, and further prolonged to 14 days with the addition of $\mathrm{CE}$ stabilizer, which reaches the standard requirement of large-scale industry manufacturing.

\section{Mechanical Properties}

Finally, tensile tests were conducted to characterize the mechanical properties of epoxy resin systems. The details of the preparation of testing specimens are described in the Supplementary Material. Mixed with required quantities of the curing agent 2-MI, EGE-2-MI, and EGE-2-MI/CE, the average value and standard deviation of the $\mathrm{Al} / \mathrm{Al}$ adhesion shear strength of the epoxy systems are shown in Figure 2F. Comparing the shear strength value of the three systems, we found that the system using the 2-MI system as a curing agent has a shear strength value of $10 \mathrm{MPa}$, while the system using the EGE-2-MI system has a relatively higher shear strength of 14.85 $\mathrm{MPa}$. We believe this can be explained by the low viscosity of the EGE-2-MI system, leading to higher wettability on the aluminum substrate and less defects in the bonding joints. A further increase to $20.13 \mathrm{MPa}$ in shear strength is observed in the system with the addition of CE stabilizer. This is because CE has an extremely high adhesion effect to metal, by bonding with hydroxyl groups on the metal surface and forming 
complex compounds with metal ions (Throckmorton et al., 2018).

Meanwhile, the tensile strength of the five samples was tested, and the averages were calculated, as shown in Figure 2F. Compared to a tensile strength at $26.78 \mathrm{MPa}$ for the system directly using $2-\mathrm{MI}$ as the curing agent, the average tensile strength of the EGE-2-MI system without or with adding stabilizer $\mathrm{CE}$ is 35.62 and $41.05 \mathrm{MPa}$, respectively. It is worth mentioning that the performance of the modified curing system is even superior to that of the unmodified epoxy system. Cyanate esters were cured via addition polymerization to produce a heterocyclic ring referred to as a polycyanurate network. This polycyanurate network is responsible for the excellent tensile properties of resulting epoxy resin systems. Since increases are observed both in shear strength and tensile strength, the conclusion that the cured EGE-2-MI/CE system has better mechanical properties can be drawn.

\section{CONCLUSION}

In conclusion, we have successfully fabricated a modified conjugated molecule EGE-2-MI. The E-51 epoxy resin can be efficiently cured by EGE-2-MI at a moderate temperature (around $110^{\circ} \mathrm{C}$ ), and the corresponding curing activation energy is $86.34 \mathrm{~kJ} / \mathrm{mol}$. The modification of $2-\mathrm{MI}$ enhanced the storage ability of the E-51 epoxy system, and the storage period of 14 days can be achieved by adding CE as a stabilizer, which reaches the standard requirement of industry manufacturing. The curing products also show promising mechanical adhesive properties, with a tensile strength of $41 \mathrm{MPa}$ and an $\mathrm{Al}-\mathrm{Al}$ shear strength of $20 \mathrm{MPa}$. We believe

\section{REFERENCES}

Acocella, M. R., Corcione, C. E., Giuri, A., Maggio, M., Maffezzoli, A., and Guerra, G. (2016). Graphene oxide as a catalyst for ring opening reactions in amine crosslinking of epoxy resins. RSC Adv. 6 (28), 23858-23865. doi:10.1039/ C6RA00485G

Arimitsu, K., Fuse, S., Kudo, K., and Furutani, M. (2015). Imidazole derivatives as latent curing agents for epoxy thermosetting resins. Mater. Lett. 161, 408-410. doi:10.1016/j.matlet.2015.08.141

Baroncini, E. A., Kumar Yadav, S., Palmese, G. R., and Stanzione, J. F. (2016). Recent advances in bio-based epoxy resins and bio-based epoxy curing agents. J. Appl. Polym. Sci. 133, 44103-44122. doi:10.1002/app.44103

Dai, Z., Li, Y., Yang, S., Zhao, N., Zhang, X., Xu, J., et al. (2009). Kinetics and thermal properties of epoxy resins based on bisphenol fluorene structure. Eur. Polym. J. 45 (7), 1941-1948. doi:10.1016/j.eurpolymj.2009.04.012

Ding, L., Yang, J. P., Chen, G., Li, H., Su, H., et al. (2017). Preparation and properties of latent moderate-temperature curing epoxy resin system. Acta Mater. Compos. Sin. 34 (10), 2150-2155. doi:10.13801/j.cnki.fhclxb.20161219.001

Fang, W., Jun, X., Jing-Wen, W., and Shu-Qin, L. (2008). A novel imidazole derivative curing agent for epoxy resin: synthesis, characterization, and cure kinetic. J. Appl. Polym. Sci. 107 (1), 223-227. doi:10.1002/app.27053

Ferdosian, F., Yuan, Z., Anderson, M., and Xu, C. (2015). Sustainable lignin-based epoxy resins cured with aromatic and aliphatic amine curing agents: curing kinetics and thermal properties. Thermochim. Acta 618, 48. doi:10.1016/j.tca.2015.09.012

Ham, Y. R., Kim, S. H., Shin, Y. J., Lee, D. H., Yang, M., Min, J. H., et al. (2010). A comparison of some imidazoles in the curing of epoxy resin. J. Ind. Eng. Chem. 16 (4), 556-559. doi:10.1016/j.jiec.2010.03.022 this novel moderate-temperature curing system with decent storage life and mechanical properties can overcome the rooted disadvantage of imidazole curing agents and open up their potential application in large-scale industry manufacturing.

\section{DATA AVAILABILITY STATEMENT}

All datasets presented in this study are included in the article/ Supplementary Material.

\section{AUTHOR CONTRIBUTIONS}

LD prepared materials and carried out experiments. XH helped analyze experimental data. TT helped characterize materials. JY supervised the work.

\section{FUNDING}

This study was financially supported by Research Projects of Shaanxi University of Technology (SLGKY2012), National Natural Science Foundation of China (21905164), and Project of Shaanxi Provincial Department of Education (17JK0140).

\section{SUPPLEMENTARY MATERIAL}

The Supplementary Material for this article can be found online at: https://www.frontiersin.org/articles/10.3389/fmats.2020.577962/ full\#supplementary-material

Hardis, R., Jessop, J. L. P., Peters, F. E., and Kessler, M. R. (2013). Cure kinetics characterization and monitoring of an epoxy resin using DSC, Raman spectroscopy, and DEA. Compos. Appl. Sci. Manuf. 49, 100-108. doi:10. 1016/j.compositesa.2013.01.021

Lee, D. H., Yang, M., Kim, S. H., Shin, M. J., and Shin, J. S. (2011). Microencapsulation of imidazole curing agents by spray-drying method. J. Appl. Polym. Sci. 122 (2), 782-788. doi:10.1002/app.34008

Leena, K., Soumyamol, P. B., Baby, M., Suraj, S., Rajeev, R., and Mohan, D. S. (2017). Non-isothermal cure and decomposition kinetics of epoxy-imidazole systems. J. Therm. Anal. Calorim. 130, 1053-1059. doi:10.1007/s10973-0176410-5

Lei, D., Ma, W., Wang, L., and Zhang, D. (2015). Preparation of 2-ethyl-4methylimidazole derivatives as latent curing agents and their application in curing epoxy resin. J. Appl. Polym. Sci. 132 (38), 423563. doi:10.1002/app.42563

Liu, L., and Li, M. (2010). Curing mechanisms and kinetic analysis of DGEBA cured with a novel imidazole derivative curing agent using DSC techniques. J. Appl. Polym. Sci. 117 (6), 3220-3227. doi:10.1002/app.32196

Maka, H., Spychaj, T., and Pilawka, R. (2012). Epoxy resin/ionic liquid systems: the influence of imidazolium cation size and anion type on reactivity and thermomechanical properties. Ind. Eng. Chem. Res. 51 (14), 5197-5206. doi:10.1021/ie202321j

Mozaffari, S. M., Beheshty, M. H., and Mirabedini, S. M. (2016). Microencapsulation of 1-methylimidazole using solid epoxy resin: study on microcapsule residence time and properties of the system. Iran Polym. J. 25 (4), 385-394. doi:10.1007/s13726-016-0430-z

Sheng, X., Akinc, M., and Kessler, M. R. (2008). Cure kinetics of thermosetting bisphenol E cyanate ester. J. Therm. Anal. Calorim. 93 (1), 77-85. doi:10.1007/ s10973-007-8803-3 
Throckmorton, J. A., Feldman, G., Palmese, G. R., Guenthner, A. J., Lamison, K. R., Redeker, N. D., et al. (2018). Hydrolytic degradation kinetics of bisphenol e cyanate ester resin and composite. Polym. Degrad. Stabil. 151, 1-11. doi:10. 1016/j.polymdegradstab.2018.02.009

Unruh, D. A., Pastine, S. J., Moreton, J. C., and Fréchet, J. M. J. (2011). Thermally activated, single component epoxy systems. Macromolecules 44 (16), 6318-6325. doi:10.1021/ma200673v

Vyazovkin, S., Chrissafis, K., Di Lorenzo, M. L., Koga, N., Pijolat, M., Roduit, B., et al. (2014). ICTAC kinetics committee recommendations for collecting experimental thermal analysis data for kinetic computations. Thermochim. Acta 590, 1-23. doi:10.1016/j.tca.2014.05.036

Wong, F. F., Chen, K.-L., Lin, C. M., and Yeh, M.-Y. (2007). New investigation of 1substituted imidazole derivatives as thermal latent catalysts for epoxy-phenolic resins. J. Appl. Polym. Sci. 104 (5), 3292-3300. doi:10.1002/app.26002

Xiong, X. H., Guo, X. C., Ren, R., Zhou, L., and Chen, P. (2019). A novel multifunctional glycidylamine epoxy resin containing phthalide cardo structure: synthesis, curing kinetics and dynamic mechanical analysis. Polym. Test. 77, 105917. doi:10.1016/j.polymertesting.2019.105917

Yang, B., Mao, Y., Zhang, Y., Bian, G., Zhang, L., Wei, Y., et al. (2019). A novel liquid imidazole-copper (II) complex as a thermal latent curing agent for epoxy resins. Polymer 178, 121586. doi:10.1016/j.polymer.2019.121586

Yang, S., Zhang, Q., Hu, Y., Ding, G., Wang, J., Huo, S., et al. (2018). Synthesis of s-triazine based tri-imidazole derivatives and their application as thermal latent curing agents for epoxy resin. Mater. Lett. 216, 127-130. doi:10.1016/j.matlet. 2017.12.122
Zeng, W., Deng, Z., Wang, H., Zhang, H., and Zhou, Q. (2019). Benzodifuranone based color-changing epoxy-polyamine coating. Dyes Pigments 164, 198-205. doi:10.1016/j.dyepig.2019.01.016

Zeng, W., Zhou, Q., Zhang, H., and Qi, X. (2018). One-coat epoxy coating development for the improvement of UV stability by DPP pigments. Dyes Pigments 151, 157-164. doi:10.1016/j.dyepig.2017.12.058

Zhang, H., Zeng, W., Du, H., Ma, Y., Ji, Z., Deng, Z., et al. (2020). Comparison for color change between benzodifuranone and benzodipyrrolidone based epoxy coating. Dyes Pigments 175, 108171. doi:10.1016/j.dyepig.2019. 108171

Zhao, X., Huang, Z., Song, P., Chen, H., Yang, H., Zhang, Y., et al. (2019) Effect of isophorone diamine on curing kinetics and mechanical properties of 2-ethyl-4methylimidazole/epoxy resin crosslinked network. Thermochim. Acta 680 (37), 178380. doi:10.1016/j.tca.2019.178380

Conflict of Interest: The authors declare that the research was conducted in the absence of any commercial or financial relationships that could be construed as a potential conflict of interest.

Copyright (c) 2020 Ding, Yang, Hao and Tong. This is an open-access article distributed under the terms of the Creative Commons Attribution License (CC $B Y)$. The use, distribution or reproduction in other forums is permitted, provided the original author(s) and the copyright owner(s) are credited and that the original publication in this journal is cited, in accordance with accepted academic practice. No use, distribution or reproduction is permitted which does not comply with these terms. 